\title{
Harbin Municipal Traffic Congestion Control Countermeasures Based on Big Data
}

\author{
Ping Liu ${ }^{1, a}$ Jia Liu ${ }^{2, b}$ \\ ${ }^{1}$ School of Finance and public management, Harbin University of Commerce, Harbin, Heilongjiang 150028, China \\ ${ }^{2}$ School of Finance and public management, Harbin University of Commerce, Harbin, Heilongjiang 150028, China \\ a781192907@qq.com \\ b972585645@qq.com
}

\begin{abstract}
Harbin municipal government fully agrees with the scientific management of traffic, constantly improves the technical level and management level, and establishes the traffic management system, which enables the traffic management department to deal with road accidents in time, and avoid traffic congestion and other traffic problems to be improved. Traffic information sharing shows the intuitiveness of data. In case of congestion, the trend of each department can be seen, which is convenient to control, adjust and record data in time, and promote the progress of cross department business collaboration. The simple information sharing of relevant departments is conducive to the coordination and management, unified planning, and avoid the serious uneven distribution of information and the game between the strong and weak departments. This paper will put forward some problems and corresponding countermeasures for the problems of traffic congestion in Harbin Based on big data.
\end{abstract}

Keywords: Intelligent transportation, traffic jam, big data

\section{INTRODUCTION}

In Harbin, some main roads prone to traffic congestion still have unreasonable planning. For example, the green belt in the middle and on both sides of the road is too wide to occupy the lane. For example, the number of traffic lights in Youyi Road section leads to long traffic time. If the traffic flow is large, especially in the morning and evening peak, Hexing road and xidazhi Street will inevitably have congestion. It takes time for the upper and lower bridge entrances and exits. In addition, in campuses and business districts, such as Tieling primary school, Fenghua middle school, high school attached to normal university and Jiaohua E-world, there are dense roads along central street, and there are always congestion conditions during the time of picking up students and commuting. In these places with high density of vehicles, roads are not planned with high quality through the big data platform. Once a large number of vehicles rush in, it will cause traffic congestion that can not be ignored.

\subsection{Traffic Congestion Control}

\subsubsection{Inconsistent direction of command and dispatch based on Internet of things platform}

Although the Internet of things platform has been initially built, different departments are responsible for different businesses, which leads to the personnel in each department can not be unified through the direction of data network command and scheduling, so that the control of traffic congestion can not achieve high efficiency and high quality. When directing the business management, monitoring and early warning, analyzing and judging of relevant departments, and dispatching human, financial, material and other resources, the smart transportation based on big data has no unified system constraints, no clear direction for decision-making, and the opinions of various departments are easy to contradict, which makes the traffic data fail to achieve the maximum benefits and fails to live up to the original intention of the establishment of the Internet of things data platform. 


\subsubsection{The integration and coordination of government data resources did not achieve high standards}

Based on big data, the Internet plus digital government integrates traffic information from related departments, vehicles, travel individuals and road strongholds, and extracts valuable data that can be further analyzed. While Harbin municipal traffic is in a mode of data integration and coordination, the transition from extensive to intensive is relatively slow. It has not yet reached a high level, so that the government in the process of data sharing, management and implementation has yet to be promoted, there is no high standard of overall coordination, leading to imperfect planning and organizational shortcomings. The unfiltered traffic data leads to information confusion, which makes it difficult to store, manage and use. The construction of digital government needs higher standard data filling in order to get through channels and deliver fresh blood. [1]

\subsubsection{The data control system lacks reasonable risk cost evaluation}

Although Harbin makes every effort to use big data intelligent system to control traffic congestion, it is a new experience and action after all. It is difficult to say that the municipal government handles every key step of the transportation system properly, including the evaluation of cost and risk in every indispensable step, such as using basic data, optimizing the internal structure of the Department, and the management and control process.

The traffic data information of Harbin smart government lacks the prediction of the material cost of equipment, talents, etc. and the time cost of precise governance before the implementation of the countermeasures, and the assessment of the possible risks after the implementation of the countermeasures. Because the development of Harbin smart data governance of traffic congestion is far from reaching the mature stage, there is no detailed and extensive risk assessment, so it is necessary to improve the governance In the process of reform, it will expose uncontrollable or avoidable problems, resulting in low efficiency and low level of government administration, resulting in irreparable losses.

\section{COUNTERMEASURE}

\subsection{Planning traffic construction through data monitoring platform}

\subsubsection{Optimize and adjust the planning and construction of important roads and bridges}

Based on the big data platform, the traffic conditions of all roads in the city are visually displayed, the congestion points are accurately warned, and the traffic data information of different intersections, trunk roads, stops and so on are analyzed, so as to provide reference for the relevant departments of Harbin to plan the construction of roads that are easy to block in each district. One or two lanes are added at the intersection of roads and bridges, or new bridges are built to reduce the traffic pressure under viaducts and disperse one lane Part of the vehicles on the old bridge to the new bridge can also control traffic congestion to a certain extent. [2] for example, in order to eliminate the blocking area of the second ring road where the number of vehicles is more than 6000 per hour in the peak period of more than 8 hours per day, we should improve the progress of the East Second Ring Road viaduct construction project, build an interchange at the intersection of Changjiang Road, connect it with the main bridge of Nanzhi Road, remove some bridges and approaches of Xianfeng Road interchange and Gongbin road viaduct, and build the roads along the viaduct Signal lights should be added to ensure that the traffic on and under the bridge can run separately without affecting each other's orderly traffic and dispersing part of the traffic flow.

\subsubsection{Guarantee the synchronous development of supporting intelligent transportation facilities}

Harbin traffic management department can use the "traffic management 12123" mobile phone app accident online processing function to send text, photos, voice and video at any time. The whole process of traffic accident processing becomes more simple, which is not only beneficial to the public to solve the accident as soon as possible, but also to improve the use of the Internet The efficiency level of traffic accidents also avoids the traffic congestion caused by the traffic accidents in this section, and improves the speed of emergency handling and the quality of traffic police handling major accidents. [3] increase the development of parking system relying on big data, so that the Internet can penetrate into the projects for the convenience of the people, so as to facilitate the people to query and reserve parking spaces online around the business districts and residential areas with large traffic flow. For example, according to the system route instructions, people can choose to enter the Sofia business district in Manhattan from Aijian Road, Jingwei street, Jiangpan Road, etc., and inform Youxing through the system around Lesong business district Park at the far end of Fulu or qiaoxia parking lot to prevent drivers from finding parking space to rush into the lane and causing difficulties.

\subsubsection{Consolidate the platform foundation of big data Internet of things}

Harbin municipal transportation departments use the Internet of vehicles to provide various detailed and accurate basic data for the prevention of traffic 
congestion, implement grid based management for information collection, reporting and monitoring, and can release road congestion status and monitor traffic accidents in real time, so as to provide big data technical support and big data control guarantee for the scientific governance of government departments. Consolidate the big data Internet of things system to achieve the goal of combining various methods from a single form, from flat research to three-dimensional comprehensive analysis, from single data to multiple data collection, from incomplete information mode to comprehensive, from overall structure management to meticulous service process management. Make full use of the Internet of things and cloud computing to deeply mine and analyze data, and provide complete, accurate and vivid data support for all departments.

\subsection{Establish the city's traffic operation command and emergency dispatching center}

\subsubsection{Forecast traffic peak and command accurately}

Based on the bus GPS, GIS, on-board video, IC card and other systems in Harbin, we can master the operation information of the bus line. For this, the traffic management department can combine the historical data to form a comparative chart and forecast data, accurately grasp the passenger flow law, and immediately issue the operation instructions, so as to avoid that each bus is sent out a lot of time apart, which can be clearly observed in the intelligent command system, Each bus represents the corresponding dot. If there is a large area of dot density around a certain location, it means that there is congestion in this area, and the command vehicle is adjusted in real time according to the situation. [4]

Through the integration of data and real-time information, it can realize the remote real-time monitoring of road traffic conditions, intuitively show the peak flow, and make the business preparations for each department in advance at the time of dense vehicles, with orderly actions and proper command. For example, at the intersection of Huanghe Road and Songshan Road, the relevant traffic data test is carried out to measure the traffic flow to the entry lane, and the corresponding passage time is allocated according to the number of cars passing in each entrance direction, so as to minimize the situation that the intersection with cars needs to stop waiting for the traffic light, and the intersection without cars needs to go through the green light.

\subsubsection{Upload congestion information for quick response}

According to the leadership of developed information technology such as big data, Internet of things and mobile computing, the flow velocity scatter diagram of frequently congested road sections is formed. It is clear whether the cause of congestion is too many vehicles or traffic accidents. It is necessary to quickly sense the road conditions and judge the traffic situation. Timely access to traffic congestion related information and high-tech induction and sorting out of macro data through the Internet of things are conducive to accelerating the next step of implementation ideas and suggestions, making comprehensive use of a variety of traffic control resources, responding to emergencies as soon as possible, reducing the impact of long congestion time on the masses and urban environment, and making administrative work more efficient and convenient, To provide technical support for government decisionmaking and organizational efficiency.

\subsection{Overall planning and coordination to realize traffic information sharing}

\subsubsection{Improve the horizontal information network chain}

The digital government based on big data is connected horizontally and vertically, the cross department efficient collaborative governance, the transportation information transmission process forms the network, breaks through the information barrier, integrates the data resources, realizes the smooth flow of data resources, enables the municipal transportation related departments to grasp the data collection, collection, sorting, sharing information, cooperation and coordination, makes the scientific decision-making, and optimizes the process If the data and service quality are not readily available, the departments will not be able to make the right decisions. [5] for example, with the cooperation of the municipal traffic management department, urban management department and Nangang District government, and the active cooperation of water supply, drainage, power and other pipeline units, the information of congested road resources was transmitted, and the scientific organization, active coordination and overall promotion were carried out, which ensured the successful construction of the interchange project around Zhongxing Avenue, Fudan street, Nankai street and Haxi street, greatly improved the traffic format and eliminated the blocking points .

\subsubsection{Realize the openness and transparency of traffic data and services}

E-government gradually makes more use of the development of big data. The municipal transportation department stores a large number of useful data closely related to the public's daily travel, understands the vast majority of the whole social data resources, and makes the data open and transparent, so as to serve the society. It also helps the government to make scientific decisions and integrate different aspects of traffic data in the 
intelligent transportation system It is an important means to solve the problem of traffic congestion by analyzing the relationship between information so that traffic services can be more targeted. The openness and transparency of transportation services enable the implementation of traffic congestion control policies in the sunshine, and let the public know the efforts made by the municipal government on the problem of congestion control. The government can evaluate the effect of government work according to the real results, win more trust, understanding and support from the people, and give the people a full sense of gain.

\subsection{Feedback control of traffic congestion}

It is very important to strengthen the evaluation of all aspects of the traffic congestion management system, establish the action reference system to improve the traffic of civilized city, and scientifically determine the evaluation content and standard according to the actual situation of Harbin. Recently reconstructed roads, urban comprehensive planning, public safety and traffic management and other departments jointly carry out project pre evaluation and post evaluation, constantly combine with the specific requirements of traffic management, comprehensively manage the impact of evaluation methods, and establish supervision and review mechanism to develop and improve the implementation of operational and technical specifications. The professional assessors of the third-party traffic management system are entrusted to conduct a comprehensive evaluation of the whole traffic system in Harbin, so that multiple departments can find the problems in the construction process of the data system, determine the development direction, and quickly adapt to the new situation and demand of big data. Harbin's traffic congestion management process enables us to evaluate whether the congestion has been effectively solved through the evaluation system, so that we can analyze the positive impact and the reasons for not getting the ideal results, which will promote the improvement of the government's further measures.

\section{CONCLUSION}

The intensive development of big data is affected by many factors, such as economic conditions, technical level of personnel and so on. The government needs to carefully plan and promote each process and node required by the intensive architecture in the four links of top-level design, step-by-step implementation, pilot promotion and continuous improvement to achieve the expected results.

On the basis of centralized data sharing, we should consolidate a convenient, practical, fast, efficient and coordinated traffic management system, strengthen the overall planning and design, increase demand, and optimize the allocation of technology, capital and personnel. Using the digital modern transportation system to save management funds, intensively control the financial investment of traffic congestion control, determine the investment scale and schedule according to the effect, and run the concept of "effect oriented" through the whole process of traffic congestion control. Intensive construction will constitute a unified supervision of operation and maintenance, centralized management of information and data, and centralized delivery of content services, so as to realize the optimization and integration of government resources, mutual recognition, standardization and coordination of management paradigm.

\section{REFERENCES}

[1] Lu, H.P., Sun, Z.Y., Qu, W.C. (2015) Big data and its application in Urban Intelligent Transportation System. Transportation system engineering and information, 15: 45-52.

[2] Guan, Z.C., Li, X., Zhang, X., Hu, B. (2012) Research on planning, design and construction management of intelligent transportation system led by government. China Public Security (Comprehensive Edition), 17: 149-158.

[3] Song, Q.W., Yang, J.F., Xie, H.T. (2014) Research on architecture and planning of Urban Intelligent Transportation System. Traffic standardization, 42: 34-39.

[4] Zhao, P.J., Li, K. (2014) Theoretical analysis of the role of big data method in alleviating urban traffic congestion. Research on modern city, 10: 25-30.

[5] Xiong, G., Dong, X.S., Zhu, F.H., Ji, T.K. (2015) Urban traffic big data technology and intelligent application system. Big data, 1: 81-96. 